Fernández-Guerrero, M., Suárez-Ramírez, M., Rojo-Ramos, J. y Feu-Molina, S. (2021). Acoso escolar en Educación Primaria y en Educación Secundaria en Badajoz: análisis de los factores de riesgo. Revista Electrónica Interuniversitaria de Formación del Profesorado, 24(2),97-111.

DOI: https://doi.org/10.6018/reifop.434581

\title{
Acoso escolar en Educación Primaria y en Educación Secundaria en Badajoz: análisis de los factores de riesgo
}

Manuel Fernández Guerrero, Miriam Suárez Ramírez, Jorge Rojo Ramos, Sebastián Feu Molina

Universidad de Extremadura

\section{Resumen}

En el periodo de la transición de Ed. Primaria a Educación Secundaria Obligatoria es donde se producen la mayoría de los casos de acoso escolar a lo largo de toda la geografía española. Es muy importante detectar dicha problemática con premura para analizarla y erradicarla de los centros escolares.

Con este estudio se ha pretendido identificar las formas de violencia que se producen en las aulas, y analizar la percepción del acoso escolar para cada una de las formas de violencia recibida y observada en función de las características de los alumnos de Ed. Primaria y ESO de la ciudad de Badajoz. Para ello han participado 1093 alumnos de $6^{\circ}$ de Ed. Primaria y de $1^{\circ}$ de Educación Secundaria Obligatoria de 19 centros educativos con edades entre 11 y 14 años. El instrumento utilizado ha sido el Cuestionario de Violencia Escolar Cotidiana (CUVECO).

Entre las conclusiones más destacadas podemos reseñar que en Badajoz existen algunos casos de acoso escolar con puntuaciones elevadas, las chicas observan más el acoso escolar, los chicos reciben más violencia verbal, la violencia observada predomina en ESO y a medida que la edad aumenta el alumno cambia la violencia verbal por la violencia física.

\section{Palabras clave}

Bullying; intimidación; educación; escolar.

\section{Bullying in Primary Education and Secondary Education in Badajoz: a risk factor analysis}

\section{Abstract}

In the period of the transition from Primary to Compulsory Secondary Education is where most cases of bullying occur throughout the Spanish geography. It is very important to detect this problem quickly to analyse it and eradicate it from schools. 
This study has been aimed at identifying the forms of violence occurring in classrooms, and analysing the perception of bullying for each of the forms of violence received and observed based on the characteristics of Primary Ed. and ESO students in the city of Badajoz. For this purpose, 1093 students from 6th Ed. Primary and 1st Compulsory Secondary Education of 19 schools between 11 and 14 years old participated. The instrument used has been the Daily School Violence Questionnaire (CUVECO).

Among the most noteworthy conclusions we can say that in Badajoz there are some cases of bullying with high scores, girls see more bullying, boys receive more verbal violence, observed violence predominates in ESO and as age increases the student changes verbal violence by physical violence.

\section{Key words}

Bullying; intimidation; education; School.

\section{Introducción}

En la última década se ha producido una mayor conciencia social sobre la problemática del acoso escolar. Los medios de comunicación tienen mucha influencia y gracias a ellos constantemente se están publicando tanto en televisión, radio, prensa y redes sociales diversos reportajes, estudios e incluso actos no gratos de sucesos de acoso escolar.

Desde la infancia tenemos que educar al escolar en valores constructivos con el fin de que cuando sea adolescente no se convierta en un alumno acosador. Actualmente, es poco frecuente encontrar un centro educativo de toda la geografía española donde nunca se haya producido algún caso de intimidación, hostigamiento, maltrato físico, verbal o psicológico o incluso abuso de poder. Muchas veces, dichos casos no son tipificados de acoso escolar porque no se producen de manera reiterada en el tiempo y con mucha frecuencia, pero otras tantas veces sí lo son, y tanto la comunidad educativa, psicólogos y orientadores de los centros educativos como familias han trabajado codo con codo para erradicar dichas conductas de las aulas escolares. Ortega y Mora-Merchán (2008) afirman que todas las conductas mencionadas anteriormente no son comportamientos puntuales ni una respuesta esporádica, sino una secuencia de acciones repetidas entre unos protagonistas, agresor/es y víctima, cuya relación persiste en el tiempo y desarrolla una determinada y conocida dinámica.

Son muchos autores los que han definido el acoso escolar. A nuestro juicio, una de las mejores definiciones de acoso escolar es la aportada por Rigby (2002) que lo define como "aquel comportamiento intencional para provocar daño, que es de carácter verbal, físico o relacional, que es repetitivo y perdura en el tiempo, en el que no media provocación y en el que existe una asimetría de poder entre el agresor y la víctima".

Según Del Rey, Ortega y Feria (2008), se entiende por factores de riesgo aquellos elementos que, en mayor o menor medida, predicen, o pueden predecir, la probabilidad de que emerja la situación o fenómeno indeseado; en este caso, la violencia. En el acoso escolar podemos encontrarnos multitud de factores de riesgos. El sexo y la edad son los dos que más predominan en la problemática del acoso escolar. El sexo masculino predomina tanto en el rol agresor como en el de víctima (Avilés, 2002; Benítez y Justicia, 2006; Hernández y Casares, 2002; Orte, Ferrá, Ballester y March, 1999; Orte, 2003; Solberg y Olweus, 2003; Tapper y Boulton, 2005) y la edad predominante de dicho acto se produce entre los 11 y los 13 años (Eslea y Rees, 2001; Garagordobil y Oñederra, 2008; Rajmil et al., 2009), aunque se está 
verificando en la última década que cada vez se aprecia este fenómeno en edades más tempranas (Cerezo, Calvo y Sánchez, 2004). Por otro lado, autores como Aguilar, Sroufe, Egeland y Carlson (2000) y Sánchez, Díaz y Valero (2014) afirman que el acoso escolar predomina en la transición de Educación Primaria a Educación Secundaria Obligatoria.

Otros factores de riesgo a tener en cuenta según Unnever y Cornell (2003) es que los sujetos inmigrantes se encuentran involucrados con mayor frecuencia como agresores mientras que Wolke, Woods, Stanford y Schulz (2001) señalan que los sujetos de otros países o razas se ven implicados como víctimas, siendo el insulto racista el tipo de agresión más padecida. Por otra parte, Pedreira, Cuesta, y Bonet (2011), Sánchez y Cerezo (2010) y Solberg y Olweus (2003) señalan que los ACNEAE, por lo general, presentan grandes problemas relacionados con la violencia y con otras conductas antisociales y según Farrington (2005); Jadue (2003); Roa, Vacas y Moreno (2006) y Silver, Meselle, Armstrong y Essex (2005) el aumento de separaciones y divorcios o el alto índice de madres solteras son factores que guardan una relación positiva con la violencia escolar.

Por otro lado, la titularidad del centro parece influir en la frecuencia del acoso. Las situaciones de acoso escolar son más frecuentes en los centros privados/concertados que en los públicos (Garaigordobil, Martínez-Valderrey, Páez y Cardozo, 2014; León del Barco, Castaño, Gómez y López Ramos,2011).

A causa de la complejidad del fenómeno, en ocasiones los cuestionarios para la evaluación de la violencia escolar se centran en múltiples aspectos, como la frecuencia de aparición, la evolución en los últimos años, los escenarios, las medidas tomadas por el profesorado, las propuestas de solución o el perfil de víctimas, agresores y espectadores, entre otros asuntos (Álvarez, Álvarez, González-Castro, Núñez y González-Pienda, 2006). Por ello, podemos encontrarnos multitud de cuestionarios que evalúan el acoso escolar. Entre los que se pueden destacar están el Cuestionario de Evaluación de la Violencia entre iguales en la Escuela y en el Ocio (CEVEO) de Díaz-Aguado, Martínez y Martín (2004), el cuestionario sobre Intimidación y Maltrato Entre Iguales (CIMEI) de Avilés (1999), el cuestionario sobre abusos entre compañeros de Fernández y Ortega (1999), el Cuestionario de estrategias de afrontamiento de la violencia escolar de Del Rey y Ortega (2005), el cuestionario sobre Problemas de la Convivencia Escolar de Peralta, Sánchez, Trianes y De la Fuente (2003) o el cuestionario de Violencia Cotidiana de Fernández-Baena, Trianes, de la Morena, Escobar, Infante y Blanca (2011) entre otros.

Debido a la alarma social creada por las situaciones de acoso en las aulas y que la comunidad autónoma de Extremadura no contempla ningún protocolo de bullying ni en la versión cibernética en su legislación Rubio-Hernández, Díaz-López y Cerezo-Ramírez (2019) se han planteado dos objetivos principalmente: identificar las formas de violencia que se producen en las aulas, y analizar la percepción del acoso escolar para cada una de las formas de violencia recibida y observada en función de las características de los alumnos de Ed. Primaria yESO.

\section{Metodología}

Se realizó una investigación con una estrategia asociativa y con un diseño de cohorte transversal (Ato, López y Benavente, 2013), donde la toma de datos se realizó mediante una encuesta. 


\section{Participantes}

En este estudio han participado 3227 escolares matriculados en $6^{\circ}$ de Ed. Primaria y $1^{\circ}$ de ESO de la ciudad de Badajoz, de los cuales un $47^{\prime} 32 \%$ estudiaban en $6^{\circ}$ de Ed. Primaria $(n=1527)$ y un $52^{\prime} 68 \%$ estudiaban en $1^{\circ}$ de E.S.O. $(n=1700)$.

La muestra de estudio final fue de 1093 alumnos de $6^{\circ}$ de Ed. Primaria y $1^{\circ}$ de Educación Secundaria Obligatoria de 19 centros educativos públicos y concertados/ privados de la ciudad de Badajoz cuyas edades oscilan entre 11-14 años $(M=11,6)$. El error de muestreo fue del $2,4 \%$ al $95 \%$ de confianza.

\section{Variables}

Las variables de estudio analizadas en este estudio fueron la percepción de la violencia escolar, la edad, el sexo, el carácter del centro educativo en el que el alumno está matriculado, curso académico, con quién vive habitualmente el alumno, número de hermanos, país de procedencia y si ha repetido algún curso.

\section{Instrumentos}

El cuestionario utilizado en esta investigación es el Cuestionario de Violencia Escolar Cotidiana (CUVECO) creado por Fernández-Baena, Trianes, de la Morena, Escobar, Infante y Blanca (2011).

El cuestionario está diseñado para evaluar la percepción de haber sufrido violencia por parte de los compañeros o de haberla observado. El cuestionario consta de 14 preguntas con respuestas de tipo Likert de cinco puntos ( $1=$ nunca y $5=$ casi siempre $)$ que hacen referencia a dos factores.

El primer factor del cuestionario CUVECO hace referencia a la experiencia personal que tiene el alumno de sufrir violencia. Se puede observar en las ocho primeras preguntas del cuestionario y evalúa las agresiones padecidas por el alumno en tres tipos: agresiones físicas, agresiones verbales y agresiones psicológicas.

El segundo factor hace referencia a la violencia observada en el centro educativo. Este factor se observa desde la pregunta número nueve hasta la pregunta número catorce. A lo largo de estas preguntas se puede evaluar los comportamientos violentos entre iguales observados por los estudiantes en el centro educativo.

El cuestionario CUVECO tiene un índice de validez de alfa de Cronbacha $=.85$ para el factor experiencia personal de sufrir violencia y $\alpha=.74$ para el factor violencia observada en el centro (Fernández-Baena, et al. 2011).

\section{Procedimiento de la toma de resultados}

El proceso de recogida de datos se inició con la selección de los centros de Educación Primaria y E.S.O. de la ciudad de Badajoz participantes en esta investigación. Para ello se contactó en primer lugar con el equipo directivo de cada uno de los centros educativos tanto públicos como concertados/ privados de la ciudad de Badajoz. Posteriormente, y una vez conocido los centros interesados en participar en el estudio, se les remitió una carta para que los padres/madres de los alumnos prestaran su consentimiento en la participación en este estudio. A continuación, se procedió a aplicar los diferentes cuestionarios en los centros educativos con la colaboración de 6 evaluadores externos debidamente entrenados.

\section{Análisis estadístico}

Finalmente se procedió a analizar los resultados estadísticos con el programa estadístico SPSS v.25 Mediante dicho programa estadístico se estudió la asociación entre las variables 
nominales a través de tablas de contingencia y los estadísticos Chi-cuadrado, V de Cramer o Coeficiente de Contingencia, analizando la asociación en las casillas de las tablas a través de los residuos tipificados corregidos (RTC).

\section{Resultados}

Inicialmente se procedió a comprobar la estructura factorial de la escala CUVECO a través de un análisis factorial exploratorio de componentes principales con rotación Oblimin. La medida de adecuación muestral fue de .878 , y la prueba de Bartlett estadísticamente significativa, $\chi_{2}(g \mid=78)=5203,80 ; p<0,01$.

En el análisis se extrajeron tres factores que explican el 39,09\%, el $12.28 \%$ y el $8,09 \%$ de la varianza, respectivamente. En la tabla 1 se puede observar que las saturaciones de cada ítem tienen pesos mayores a .50. El primer factor indica comportamientos violentos padecidos físicamente por los escolares; el factor dos indica la violencia observada en los iguales, mientras que el factor tres presenta ítems de violencia recibida verbalmente. El análisis de fiabilidad muestra valores adecuados para los tres factores, siendo superiores a .70.

Tabla 1.

Análisis factorial del cuestionario CUVECO

\begin{tabular}{lccc}
\hline & Factor 1 & Factor 2 & Factor 3 \\
\hline Me han golpeado con algún objeto &, 781 &,- 289 &,- 309 \\
Me han robado algún objeto &, 750 &,- 345 &,- 309 \\
Me han roto cosas &, 637 &,- 422 &,- 406 \\
Me han dado puñetazos o patadas &, 598 &,- 221 &,- 541 \\
Me han empujado &, 505 &,- 469 &,- 415 \\
Los alumnos se meten en peleas &, 388 &,- 795 &,- 214 \\
Los alumnos destrozan cosas &, 399 &,- 779 & \\
Los alumnos roban cosas &, 403 &,- 746 & \\
Los alumnos amenazan a otros alumnos &, 209 &,- 745 &,- 547 \\
Se dicen palabras malsonantes & &,- 731 &,- 401 \\
Me han amenazado &, 356 &,- 287 &,- 842 \\
Me han dicho que me iban a pegar &, 441 &,- 294 &,- 806 \\
Se han burlado de mí o me han despreciado &, 297 &,- 273 &,- 764 \\
\hline Alpha de Crombach & .74 & .81 & .78 \\
\hline
\end{tabular}

La prueba de Kolmogorov Smirnov indicó que las variables objeto de estudio no se cumplían el supuesto de normalidad por lo que se decidió utilizar pruebas no paramétricas. En el estudio correlacional de las formas de violencia, la prueba de correlación de Spearman indica que las tres formas de violencia están significativamente correlacionadas de forma positiva. Existe una correlación moderada entre la violencia observada y la violencia física ( $r s=.51$; $\mathrm{p}=0.00)$ y entre la violencia observada y la violencia verbal ( $r s=.407 ; \mathrm{p}=0.00)$. También se puede observar una correlación moderada entre la violencia física y la violencia verbal ( $r s=$ $.0545 ; p=0.00)$.

Si observamos dichas correlaciones, controlando el sexo y la edad, los resultados del cuestionario CUVECO indican que existe una correlación moderada entre la violencia observada y la violencia física ( $r s=.55 ; \mathrm{p}=0.00$ ) y entre la violencia observada y la violencia 
verbal ( $\mathrm{rs}=.421 ; \mathrm{p}=0.00$ ). También se puede observar una correlación moderada entre la violencia física y la violencia verbal ( $r s=.0577 ; p=0.00)$.

Tabla 2.

Correlaciones de Spearman de las formas de violencias.

\begin{tabular}{cccccccc}
\hline & & $\begin{array}{c}\text { Violencia } \\
\text { Observada }\end{array}$ & $\begin{array}{c}\text { Violencia } \\
\text { física }\end{array}$ & $\begin{array}{c}\text { Violencia } \\
\text { verbal }\end{array}$ & $\begin{array}{c}\text { Violencia } \\
\text { Observada }+\end{array}$ & $\begin{array}{c}\text { Violencia } \\
\text { física }+\end{array}$ & $\begin{array}{c}\text { Violencia } \\
\text { verbal+ }\end{array}$ \\
\hline \multirow{2}{*}{ Violencia } & $\mathrm{r}_{\mathrm{s}}$ & 1,000 & & & 1,000 & & \\
Observada & $\mathrm{p}$ & $\cdot$ & & &. & & \\
& $\mathrm{N}$ & 1093 & & & 0 & & \\
& $\mathrm{r}_{\mathrm{s}}$ &, $511^{* *}$ & 1,000 & &, 551 & 1,000 & \\
Violencia física & $\mathrm{p}$ &, 000 &. & &, 000 &. & \\
& $\mathrm{~N}$ & 1093 & 1093 & & 1089 & 0 & \\
Violencia & $\mathrm{r}_{\mathrm{s}}$ &, $407^{* *}$ &, $545^{* *}$ & 1,000 &, 421 &, 577 & 1,000 \\
verbal & $\mathrm{P}$ &, 000 &, 000 &. &, 000 &, 000 &. \\
& $\mathrm{~N}$ & 1093 & 1093 & 1093 & 1089 & 1089 & 0 \\
\hline
\end{tabular}

+ Correlaciones controlando para el sexo y edad.

Los descriptivos indican que los alumnos manifiestan que hay una mayor violencia observada $(M=2.44 \pm .890)$ en los iguales que la violencia física $(M=1.74 \pm .790)$ y la violencia verbal $(M=2.12 \pm .720)$.

Los gráficos boxplots muestran que hay casos con puntuaciones extremas de violencia percibida y recibida. En el gráfico de boxplots sobre las formas de violencia se observa que hay 17 alumnos que reciben violencia física (Figura 1), 9 alumnos de EP ( 3 chicos y 6 chicas) y 8 de ESO (2 chicos y 6 chicas); 18 alumnos que reciben violencia verbal (Figura 2), 9 de EP y 9 de ESO ( 2 chicos y 7 chicas en ambas etapas educativas); y 4 alumnos obtienen puntuaciones elevadas de la violencia observada (Figura 3), 4 de EP (2 chicos y 2 chicas) y ninguno de ESO. Observando estos gráficos, se puede ver cómo hay cuatro alumnos que reciben acoso escolar tanto físico como verbal (chica 993 con 12 años, matriculada en $1^{\circ}$ de ESO en centro concertado; chicos 624 y 1041 de 12 años, matriculados en $1^{\circ}$ de ESO de centro público y chico de 653 de 12 años matriculado en $1^{\circ}$ de ESO en centro concertado).

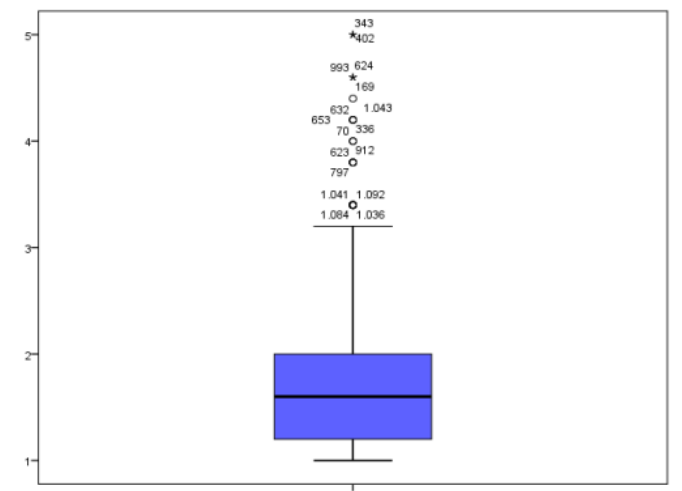

Figura 1. Gráficos boxplots de la violencia física recibida. 


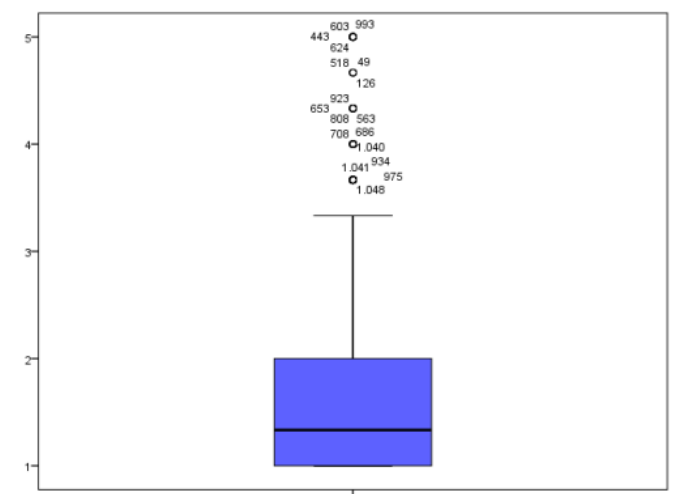

Figura 2. Gráficos boxplots de la violencia verbal recibida.

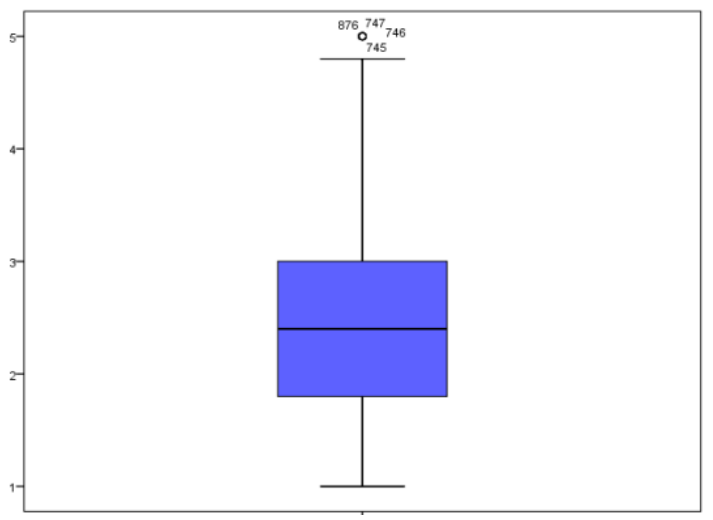

Figura 3. Gráficos boxplots de la violencia observada en los iguales.

Se realizó un análisis inferencial en la violencia observada, en la violencia verbal recibida y en la violencia física recibida, en función de las variables sociodemográficas y contextuales del centro educativo.

En la tabla 3 se presentan los resultados del análisis descriptivo e inferencial de la violencia observada. Se observan diferencias significativas en la violencia observada en función del sexo, oferta educativa y curso académico $(p<.01)$, también se encontraron diferencias en función de la edad y de con quién vive el alumno ( $p<.05)$.

Tabla 3.

Análisis descriptivo e inferencias de la violencia observada.

\begin{tabular}{|c|c|c|c|c|c|c|c|c|}
\hline & $n$ & $M$ & $\begin{array}{l}\text { Rango } \\
\text { Prom. }\end{array}$ & $U$ & $p$ & $H$ & $g l$ & $p$ \\
\hline Sexo & & & & 164285,00 & $.004^{* *}$ & - & - & - \\
\hline Hombre & 550 & 2,34 & 519,80 & & & & & \\
\hline Mujer & 543 & 2,54 & 574,55 & & & & & \\
\hline Edad & & & & & & 10,097 & 3 & ,018* \\
\hline 11 & 434 & 2,35 & 514,20 & & & & & \\
\hline 12 & 464 & 2,47 & 555,27 & & & & & \\
\hline 13 & 164 & 2,57 & 598,60 & & & & & \\
\hline 14 & 30 & 2,55 & 593,27 & & & & & \\
\hline Tipo de Centro & & & & 143695 & 0.400 & & & \\
\hline Público & 687 & 2,41 & 540,84 & & & & & \\
\hline Concertado/Privado & 406 & 2,49 & 557,43 & & & & & \\
\hline
\end{tabular}




\begin{tabular}{|c|c|c|c|c|c|c|c|c|}
\hline Oferta de niveles & & & & & & 9,751 & 2 &, $008^{*}$ \\
\hline Solo Primaria & 391 & 2,33 & 509,18 & & & & & \\
\hline Solo ESO & 294 & 2,51 & 581,85 & & & & & \\
\hline Ambas opciones & 408 & 2,49 & 558,13 & & & & & \\
\hline Curso & & & & 128350,00 & $\begin{array}{c}.000 * \\
*\end{array}$ & & & \\
\hline $6^{\circ}$ Primaria & $\begin{array}{r}62 \\
0\end{array}$ & 2,36 & 517,52 & & & & & \\
\hline $1^{\circ} \mathrm{ESO}$ & $\begin{array}{r}47 \\
3\end{array}$ & 2,54 & $\begin{array}{r}585,6 \\
5\end{array}$ & & & & & \\
\hline ¿Con quién vives? & & & & & & 9,968 & 4 & ,041* \\
\hline Con mis padres & $\begin{array}{r}91 \\
3\end{array}$ & 2,41 & 536,81 & & & & & \\
\hline Con mi padre & 21 & 2,23 & 488,19 & & & & & \\
\hline Con mi madre & 114 & 2,68 & 620,33 & & & & & \\
\hline Otros familiares & 9 & 2,22 & 500,17 & & & & & \\
\hline Otros & 6 & 2,53 & 614,00 & & & & & \\
\hline$N^{\circ}$ hermanos & & & & & & 3,064 & 3 & 0,382 \\
\hline No tengo hermanos & 123 & 2,31 & 502,62 & & & & & \\
\hline Un hermano & 60 & 2,45 & 548,4 & & & & & \\
\hline & 9 & & 0 & & & & & \\
\hline Dos hermanos & 24 & 2,48 & 558,34 & & & & & \\
\hline Tres o más hermanos & $\begin{array}{r}5 \\
116 \\
\end{array}$ & 2,46 & 562,76 & & & & & \\
\hline País de procedencia & & & & & & 2,916 & 4 &, 572 \\
\hline España & 102 & 2,45 & 549,51 & & & & & \\
\hline & 5 & & & & & & & \\
\hline Europa & 32 & 2,37 & 508,14 & & & & & \\
\hline Asia & 12 & 2,42 & 556,54 & & & & & \\
\hline África & 5 & 1,88 & 334,70 & & & & & \\
\hline América & 19 & 2,38 & 526,63 & & & & & \\
\hline ¿Has repetido algún curso? & & & & 63122,00 & .740 & & & \\
\hline $\mathrm{Si}$ & 134 & 2,48 & 555,44 & & & & & \\
\hline No & $\begin{array}{r}95 \\
9\end{array}$ & 2,44 & 545,82 & & & & & \\
\hline
\end{tabular}

Nota: $n=$ muestra: $M=$ Media; $U=$ Prueba $U$ de Mann-Whitney; $p=$ nivel de significancia; $H=$ Prueba de Kruskal-Wallis; gl=Grados de libertad.

En la tabla 4 se presentan los resultados del análisis descriptivo e inferencial de la violencia verbal recibida. Se observan diferencias significativas en la violencia observada en función de la edad, curso académico y dependiendo del número de hermano que tenga el alumno $(p<.01)$, también se encontraron diferencias en función del sexo y oferta educativa $(p<.05)$.

Tabla 4.

Análisis descriptivo e inferencias de violencia verbal recibida.

\begin{tabular}{lcccccccc}
\hline & $n$ & $M$ & $\begin{array}{l}\text { Rango } \\
\text { Prom. }\end{array}$ & $U$ & $p$ & $H$ & $g l$ & $p$ \\
\hline $\begin{array}{l}\text { Sexo } \\
\text { Hombre }\end{array}$ & 550 & 1,70 & 565,29 & & 139268,00 & $.046^{*}$ & & \\
Mujer & 543 & 1,60 & 528,48 & & & & & \\
\hline Edad & & & & & & 15,159 & 3 &, $002^{* *}$ \\
11 & 434 & 1,75 & 580,48 & & & & &
\end{tabular}




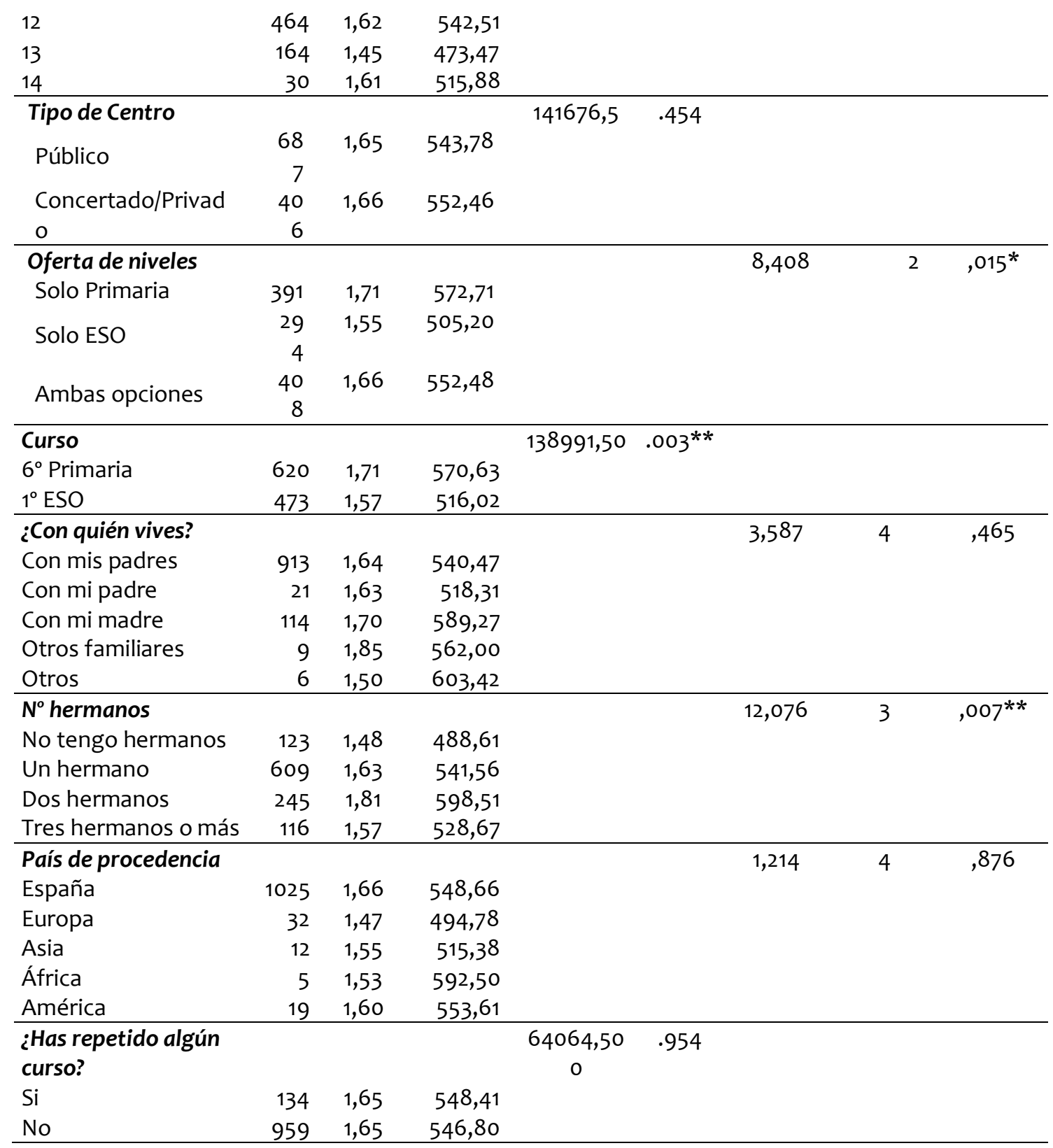

En la tabla 5 se presentan los resultados del análisis descriptivo e inferencial de la violencia física recibida. Se observan diferencias significativas en la violencia observada en función del carácter del centro educativo y dependiendo de la oferta educativa del centro escolar $(p<.05)$.

Tabla 5.

Análisis descriptivo e inferencias de violencia física recibida.

\begin{tabular}{lcccccccc}
\hline & $\mathrm{n}$ & $\mathrm{M}$ & $\begin{array}{l}\text { Rango } \\
\text { Prom. }\end{array}$ & $\mathrm{U}$ & $p$ & $H$ & gl & $p$ \\
\hline Sexo & & & & 152900,50 & .490 & & & \\
Hombre & 550 & 1,67 & 540,50 & & & & & \\
Mujer & 543 & 1,69 & 553,58 & & & 7,313 & 3 &, 063 \\
\hline Edad & & & & & & &
\end{tabular}




\begin{tabular}{|c|c|c|c|c|c|c|c|c|c|}
\hline 12 & 464 & 1,69 & 552,09 & & & & & & \\
\hline 13 & 164 & 1,58 & 507,60 & & & & & & \\
\hline 14 & 30 & 1,47 & 439,98 & & & & & & \\
\hline Tipo de Centro & & & & 149860 & $.038 *$ & & & & \\
\hline Público & 687 & 1,66 & 531,86 & & & & & & \\
\hline Concertado & 406 & 1,71 & 572,61 & & & & & & \\
\hline $\begin{array}{l}\text { Oferta de } \\
\text { niveles }\end{array}$ & & & & & & 6,986 & & 2 &, $030 *$ \\
\hline Solo Primaria & 391 & 1,68 & 546,90 & & & & & & \\
\hline Solo ESO & 294 & 1,63 & 510,28 & & & & & & \\
\hline $\begin{array}{l}\text { Ambas } \\
\text { opciones }\end{array}$ & 408 & 1,71 & 573,56 & & & & & & \\
\hline Curso & & & & 138991,50 & .136 & & & & \\
\hline $6^{\circ}$ Primaria & 620 & 1,70 & 559,32 & & & & & & \\
\hline $1^{\circ} \mathrm{ESO}$ & 473 & 1,56 & 530,85 & & & & & & \\
\hline ¿Con quién vives? & & & & & & 5,694 & 4 & & ,223 \\
\hline $\begin{array}{l}\text { Con mis } \\
\text { padres }\end{array}$ & 913 & 1,67 & 542,12 & & & & & & \\
\hline Con mi padre & 21 & 1,66 & 457,52 & & & & & & \\
\hline Con mi madre & 114 & 1,74 & 580,82 & & & & & & \\
\hline $\begin{array}{l}\text { Otros } \\
\text { familiares }\end{array}$ & 9 & 1,64 & 592,67 & & & & & & \\
\hline Otros & 6 & 1,93 & 722,42 & & & & & & \\
\hline$N^{\circ}$ hermanos & & & & & & 6,792 & 3 & & ,079 \\
\hline $\begin{array}{l}\text { No tengo } \\
\text { hermanos }\end{array}$ & 123 & 1,61 & 521,26 & & & & & & \\
\hline Un hermano & 609 & 1,71 & 568,12 & & & & & & \\
\hline Dos hermanos & 245 & 1,66 & 528,37 & & & & & & \\
\hline $\begin{array}{l}\text { Tres } \\
\text { hermanos o } \\
\text { más }\end{array}$ & 116 & 1,61 & 502,74 & & & & & & \\
\hline $\begin{array}{l}\text { País de } \\
\text { procedencia }\end{array}$ & & & & & & 4,999 & 4 & & ,287 \\
\hline España & 1025 & 1,67 & 543,11 & & & & & & \\
\hline Europa & 32 & 1,80 & 626,03 & & & & & & \\
\hline Asia & 12 & 1,58 & 505,38 & & & & & & \\
\hline África & 5 & 1,88 & 750,30 & & & & & & \\
\hline América & 19 & 1,60 & 596,71 & & & & & & \\
\hline $\begin{array}{l}\text { ¿Has repetido } \\
\text { algún curso? }\end{array}$ & & & & 63802,00 & .894 & & & & \\
\hline $\mathrm{Si}$ & 134 & 1,67 & 550,37 & & & & & & \\
\hline No & 959 & 1,68 & 546,53 & & & & & & \\
\hline
\end{tabular}

\section{Discusión de resultados}

El primer objetivo de este estudio fue identificar las formas de violencia que se producen en las aulas. El análisis factorial exploratorio mostró una estructura diferente al modelo teórico de la escala (Fernández-Baena, et al. 2011), en este estudio el factor que discriminaba la violencia recibida se dividió en dos factores, violencia física y violencia verbal.

En la ciudad de Badajoz se observan algunos casos de acoso en la edad escolar, aproximadamente un 1,5\% (17 alumnos que reciben violencia física, 9 en EP y 8 en ESO; 18 alumnos que reciben violencia verbal, 9 EP y 9 ESO; y 4 alumnos de EP observan la violencia) presentan puntuaciones por encima del tercer cuartil, véanse gráficos boxplot. Debemos de 
tener en cuenta que los alumnos que lo padecen obtienen puntuaciones elevadas en algunas de sus formar (violencia observada, violencia verbal recibida y violencia física recibida) o incluso en varias de ellas

En estudios nacionales realizados por Serrano e Iborra (2005) se observaron que el 7,6\% de los alumnos encuestados eran agresores y el 14,5\% eran víctimas. Por otro lado, en el Informe Cisneros XII sobre la Violencia y el Acoso Escolar de Oñate y Piñuel (2007) se observó cómo el $26 \%$ de los chicos y el $13 \%$ de las chicas son agresores mientras que un $24 \%$ de los encuestados eran víctimas del acoso escolar. Por ello, podemos decir que en la ciudad de Badajoz existe menos acoso escolar en comparación con los resultados obtenidos en otros estudios del territorio nacional

También se puede observar en este estudio que sí se produce más el acoso verbal en $6^{\circ}$ de Ed. Primaria que en $1^{\circ}$ de ESO, pero en el acoso físico no se observan diferencias predominantes entre un curso u otro. Algunos estudios reflejan resultados diferentes a los de este estudio, afirmando que existe mayor acoso escolar en Ed. Secundaria que en Ed. Primaria (Aguilar, Sroufe, Egeland y Carlson, 2000; Sánchez, Díaz y Valero, 2014).

En los escolares de Badajoz se registra mayor violencia observada en ESO, mientras que se manifiesta una mayor percepción de acoso verbal en Educación Primaria. En esta línea, autores como Ortega, Mora-Merchán y Mora (1995); Oñate y Piñuel (2007) y Sánchez y Ortega (2010) manifiestan que el número de víctimas del acoso escolar disminuye a medida que aumenta el curso escolar.

En la relación al segundo objetivo, se ha analizado la percepción del acoso escolar, para cada una de las formas de violencia física, verbal y observada, en función de las características de los alumnos de Educación Primaria y ESO.

La percepción de la violencia física es mayor en centros concertados, difiriendo de los resultados obtenidos por Del hoyo (2015) dónde no hay diferencias del acoso escolar en los centros públicos y concertados/ privados, y en centros dónde la oferta educativa está compuesta por Ed. Primaria y ESO. No se ha constatado que los chicos sufran más violencia física que las chicas al contrario de lo que sucede en las investigaciones llevadas a cabo por Owens, Daly y Slee (2005). Autores cómo Solberg y Olweus (2003) y Tapper y Boulton (2005) manifiestan que son los chicos los que se ven envueltos con mayor frecuencia en el fenómeno del acoso escolar, tanto en el rol de agresor como en el rol de víctima.

Por otra parte, la percepción de la violencia verbal es mayor entre el alumnado masculino de 11 años al contrario de lo que manifestaba Solberg y Olweus (2003), de $6^{\circ}$ de Educación Primaria, en centros dónde solo se imparte Ed. Primaria y que tenga dos hermanos.

$Y$ finalmente, la percepción de la violencia observada predomina entre las chicas, en centros dónde solo se imparte ESO, en $1^{\circ}$ de ESO (Aguilar, et al. 2000; Sánchez, et al. 2014), que vivan con su madre únicamente y tengan 13 años. Igualmente, González, Postigo, Montoya y Martorel (2007) y Ortega (2008) afirman que la proporción de chicas en las situaciones de acoso escolar se ha duplicado en los últimos años e, incluso, se ha igualado al de los chicos.

También se puede observar cómo nacer fuera de España, ser alumno repetidor o tener familia monoparental no implica recibir más acoso escolar, al contrario de lo que manifestaban autores como Hay, Pawlby, Angold, Harold y Sharp (2003); Flouri y Buchanan (2003), Unnever y Cornell (2003); Wolke, Woods, Stanford y Schulz (2001); y a diferencia de lo que enunciaban Díaz-Aguado (2005); Farrington (2005); Silver, Meselle, Armstrong y Essex (2005) y Roa, Vacas y Moreno (2006).

Según los resultados obtenidos, no existe asociación significativa entre la violencia verbal según el carácter del centro educativo donde está matriculado el alumno, pero sí existe diferencia significativa en la violencia física, ya que se produce más en los colegios 
concertados/ privados que en los centros públicos al igual que manifiestan Garaigordobil, Martínez-Valderrey, Páez y Cardozo, 2014; León del Barco, Castaño, Gómez y López Ramos,2011

Para finalizar, en los centros donde hay tanto Ed. Primaria como ESO sí se produce más violencia física, pero no más violencia verbal. Se puede constatar que la violencia verbal se produce en mayor proporción en los centros donde solo hay Ed. Primaria.

\section{Conclusiones}

Las conclusiones más destacadas de este trabajo de investigación son:

- En la ciudad de Badajoz existen algunos casos de acoso escolar, pero los alumnos que lo padecen obtienen puntuaciones elevadas en algunas de sus formar (violencia observada, violencia verbal recibida y violencia física recibida) o incluso en varias de ellas. Se ha podido observar que hay 17 alumnos que reciben violencia física, entre los cuales 5 de ellos obtienen puntuaciones muy altas de violencia física recibida. Por otro lado, se observan 18 alumnos que reciben violencia verbal, entre los cuales 4 de ellos obtienen puntuaciones muy altas de violencia verbal. Sin embargo, solamente se han detectado 4 alumnos con puntuaciones elevadas de violencia observada.

- Las chicas observan con más frecuencia el acoso escolar que los chicos. Por otro lado, los chicos reciben más violencia verbal que las chicas. No se observaron diferencias entre el sexo en cuanto a la violencia física recibida.

- Los alumnos de 11 años observan menos violencia escolar que los alumnos de 13 años. Sin embargo, ocurre lo contrario en la violencia verbal, ya que son los alumnos de 11 años quienes obtienen mayores puntuaciones de violencia verbal recibida que los alumnos de 13 años. Tampoco se observaron diferencias significativas en cuanto a la edad en la violencia física recibida. A modo de resumen se puede afirmar que, según los resultados obtenidos en este estudio, a medida que el aumenta la edad el alumno cambia la violencia verbal por la violencia física. Es decir, en los últimos cursos de Ed. Primaria predomina más la violencia verbal mientras que en los cursos de la ESO predomine la violencia física entre los iguales.

- En los centros concertados/ privados se produce más violencia física que en los centros públicos. Sin embargo, no se encontraron diferencias significativas entre la violencia observada y la violencia verbal en función del carácter del centro educativo.

- La violencia observada obtiene mayores puntuaciones en los centros donde solo se imparte ESO. Sin embargo, la violencia verbal predomina en los centros donde solo hay Ed. Primaria. Por otro lado, la violencia física predomina en los centros donde se imparte tanto Ed. Primaria y ESO que en los centros donde solo se imparte una etapa educativa puede deberse a dos motivos principalmente. En primer lugar, porque existe una gran masificación de alumnos matriculados en los centros donde se imparte ambas etapas educativas. En segundo lugar, debido a que los alumnos de cursos superiores tienen la tendencia de imponerse físicamente a los alumnos de menor edad con tal de mostrar su mayor jerarquía de edad y su ego.

- La violencia verbal obtiene puntuaciones más elevadas en los alumnos que cursan $6^{\circ}$ de Ed. Primaria; sin embargo, la violencia observada obtiene mayores puntuaciones en los alumnos matriculados en $1^{\circ}$ de ESO. Por otro lado, no se observaron diferencias significativas en cuanto a la violencia física y al curso donde re realice.

- Ser alumno extranjero o repetidor no es un factor de riesgo para estar más implicados en las situaciones de acoso escolar. 
- Los alumnos con familias monoparentales perciben de mejor manera la violencia observada que los alumnos que viven con ambos padres. No se detectaron diferencias significativas entre tener familia monoparental y recibir violencia física o verbal.

\section{Referencias}

Aguilar, B., Sroufe, L. A., Egeland, B. y Carlson, E. (2000). Distinguishing the earlyonset/abelleda and adolescence-onset antisocial behavior types: from birth to 16 years. Development and Psychopathology, 12, 109-132.

Álvarez, L., Álvarez, D., González-Castro, P., Núñez, J.C. y González-Pienda, J.A. (2006). Evaluación de los comportamientos violentos en los centros educativos. Revista Psicothema, 18(4), 686-695.

Ato, M., López, J. J. y Benavente, A. (2013). Un sistema de clasificación de los diseños de investigación en psicología. Anales de Psicología, 29(3), 1038-1059.

Avilés, J.M. (1999). CIMEI. Cuestionario sobre intimidación y maltrato entre iguales. Valladolid: Autor

Avilés, J.M. (2002). La intimidación y el maltrato entre iguales (bullying) en la Educación Secundaria Obligatoria. Validación del cuestionario CIMEl y estudio de incidencia. Tesis doctoral no publicada. Universidad de Valladolid, Facultad de Psicología.

Benítez, J. L. y Justicia F. (2006) El maltrato entre iguales: descripción y análisis del fenómeno. Rev. Electron Investig Psicoeduc Psicopedag 4, 151-70.

Cerezo, F., Calvo, A. y Sánchez, C. (2004). Bullying y estatus social en el grupo-aula en una muestra de escolares. IV Congreso Internacional de Psicología y Educación. Almería: Actas del Congreso.

Del hoyo, M. (2015). Estudio comparativo del acoso escolar en centros laicos y religiosos de la provincia de Málaga en 2012. Boletín criminológico. Instituto andaluz interuniversitario de criminología, 155, 6

Del Rey, R. y Ortega, R. (2005). Violencia interpersonal y gestión de la disciplina. Un estudio preliminar. Revista Mexicana de Investigación Educativa, 10(26), 805-832

Del Rey, R., Ortega, R. y Feria, I. (2008). Riesgos de la violencia escolar. Revista Informe de Investigaciones Educativas, 12(2), 257-273.

Díaz-Aguado, M. J. (2005). La violencia entre iguales en la adolescencia y su prevención desde la escuela. Psicothema, 17(4), 549-558.

Díaz-Aguado, M.J., Martínez, R., y Martín, G. (2004). Prevención de la violencia y lucha contra la exclusión desde la adolescencia. La violencia entre iguales en la escuela y en el ocio (vol. 1). Madrid: Instituto de la Juventud

Eslea, M. y Rees, J. (2001). At what age are children most likely to be bullied at school? Aggressive Behavior, 27(6), 419-429.

Farrington, D. P. (2005). Childhood origins of antisocial behavior. Clinical Psychology y Psychotherapy, 12(3), 177-190.

Fernández, I. y Ortega, R. (1999). Cuestionario sobre abusos entre compañeros. En I. Fernández (ed.): Prevención de la violencia y resolución de conflictos. El clima escolar como factor de calidad (pp. 210-219). Madrid: Narcea 
Fernández-Baena, F. J., Trianes, M. V., de la Morena, M. L, Escobar, M., Infante, L. y Blanca, M. J. (2011). Propiedades psicométricas de un instrumento para la evaluación de la violencia cotidiana entre iguales en el contexto escolar. Revista Anales de Psicología. 27(1), $102-108$.

Flouri, E. y Buchanan, A. (2003). The role of mother involvement and father involvement in adolescent bullying behavior. Journal Interpersonal Violence, 18(6), 634-644.

Garaigordobil, M., Martínez-Valderrey, V., Páez, D., y Cardozo, G (2014). Bullying y ciberbulling: diferencias entre colegios públicos-privados y religiosos y laicos. Pensamiento Psicológico, 13(1), 39-54.

Garaigordobil, M., y Oñederra, J.A. (2008). Estudios epidemiológicos sobre la incidencia del acoso escolar e implicaciones educativas. Información Psicológica, 94, 14-35.

González, R, Postigo, S. Montoya, I. y Martorell, M. C. (2007). Implicación femenina en el acoso escolar. En Situación actual y características de la violencia escolar, Vol. I, (179-184). Almería: Grupo Editorial Universitario.

Hay, D. F., Pawlby, S., Angold, A., Harold, G. T. y Sharp, D. (2003). Pathways to violence in the children of mothers who were depressed postpartum. Developmental Psychology, 39(6), 1083-1094.

Hernández, T. y Casares, E. (2002). Aportaciones Teórico-Prácticas para el Conocimiento de Actitudes Violentas en el Ámbito Escolar. Pamplona: Instituto Navarro de la Mujer.

Jadue, G. (2003). Transformaciones familiares en Chile: riesgo creciente para el desarrollo emocional, psicosocial y la educación de los hijos. Estudios pedagógicos, 29, 115-126.

León del Barco, Castaño, Gómez y López Ramos (2011). Acoso escolar en la Comunidad de Extremadura vs. Informe Español del Defensor del Pueblo (2006). Electronic Journal of Research in Educational Psychology, 9(24), 565-586.

Oñate, A. y Piñuel, I. (2007). Informe Cisneros X. Acoso y Violencia Escolar en España. Instituto de Innovación Educativa y Desarrollo Directivo.

Orte, C. (2003). Los problemas de la convivencia en las aulas. Análisis del bullying. Revista Electrónica Interuniversitaria de Formación del Profesorado, 6. http://213.0.8.18/portal/Educantabria/RECURSOS/Materiales/Biblestinv/Baleares_Pro bl_convivencia_Analisis_Bullying.pdf

Orte, C., Ferrá, P., Ballester, L. y March, M.X. (1999). Resultados de la investigación sobre bullying en la Comunidad Autónoma de las Islas Baleares. Palma de Mallorca: Mimeo.

Ortega, R. (2008). Malos tratos entre escolares: de la investigación a la intervención. Madrid: CIDE, D.L.

Ortega, R. y Mora-Merchán, J. (2008). Las redes de iguales y el problema del acoso escolar. Infancia y Aprendizaje, 4, 515-528.

Ortega, R., Mora-Merchán, J.A. y Mora, J. (1995). Cuestionario sobre intimidación y maltrato entre iguales. Proyecto Sevilla Anti-Violencia Escolar. Sevilla: Universidad de Sevilla.

Oweus, L., Daly, A y Slee, P. (2005). Sex and age differences in victimization and conflicto resolution among adolescents in a South Australian School. Aggressive behavior, 31, 112.

Pedreira, P., Cuesta, B., y Bonet, C. (2011). Acoso escolar. Pediatría Atención Primaria, 13(52), 661-670. 
Peralta, J., Sánchez, M.D., Trianes, M.V., y De la Fuente, J. (2003). Estudio de la validez interna y externa de un cuestionario sobre conductas problemáticas para la convivencia según el profesor. Psicología, Saúde \& Doenças, 4(1), 83-96.

Rajmil, L, Alonso, J. Analitis, F., Detmar, S., Erhart, M., Klein, M., Ravens-Sieberer, U., Herdman, M., Berra, S., y European Kidcreen Group. (2009). Víctimas de acoso: factores asociados en niños y adolescentes de 8 -18 años de edad en 11 países europeos. Pedriatrics (Ed esp), 67, 111-118.

Rigby, K. (2002). A meta-evaluation of methods and approaches to reducing bullying in preschools and in early primary school in Australia. Australia: National Crime Prevention (Commonwealth Attorney General's Department).

Roa, J. M., Vacas, M. D. C. y Moreno, M. (2006). Victimización escolar y clima socio-familiar. Revista Iberoamericana De Educación, 41 (1), 17-21.

Rubio Hernández, F. J., Díaz López, A., \& Cerezo Ramírez, F. (2019). Bullying y cyberbullying: la respuesta de las comunidades autónomas. Revista Electrónica Interuniversitaria De Formación Del Profesorado, 22(1). 145-157.

Sánchez, B. J., Díaz, A. y Valero, A. (2014). Mejora de la convivencia escolar a través de la Educación Física. Saarbrücken: Editorial Académica Española.

Sánchez, C., y Cerezo, F. (2010). Variables personales y sociales relacionadas con la dinámica del bullying en escolares de Educación Primaria. Electronic Journal of Research in Educational Psychology, 8(3), 1696-2095.

Sánchez, V. y Ortega, R. (2010). El estudio científico del fenómeno bullying. En R. Ortega (Coord.), Agresividad injustificada, bullying y violencia escolar, pp. 55-80. Madrid: Alianza Editorial.

Serrano, A. e Iborra, I. (2005). Informe: Violencia entre compañeros en la escuela. Centro Reina Sofía para el Estudio de la Violencia.

Silver, R. B., Measelle, J. R., Armstrong, J. M. y Essex, M. J. (2005). Trajectories of classroom externalizing behavior: Contributions of child characteristics, family characteristics, and the teacher-child relationship during the school transition. Journal of School Psychology, 43(1), 39-60.

Solberg, M. E. y Olweus, D. (2003). Prevalence estimation of school bullying with the Olweus Bully/Victim questionnaire. Aggressive Behavior, 29(3), 239-268.

Tapper, K. y Boulton, M. J. (2005). Sex differences in levels of physical, verbal, and indirect aggression amongst primary school children and their associations with beliefs about aggression. Aggressive Behavior, 30(2), 123-145.

Unnever, J. D. y Cornell, D. G. (2003). Bullying, self-control, and ADHD. Journal Interpersonal Violence, 18(2), 129-147.

Wolke, D., Woods, S., Stanford, K. y Schulz, H. (2001). Bullying and victimization of primary school children in England and Germany: Prevalence and school factors. British Journal of Psychology, 92 (4), 673. 